\title{
Analysis of Non-minimum Phase in Buck-Boost Converter
}

\author{
Huang Jinfeng ${ }^{1,2, a}$ and Liu Shulin ${ }^{1}$ \\ ${ }^{1}$ Xi'an University of Science \& Technology, 710054 Xi'an, China \\ ${ }^{2}$ Shaanxi University of Technology, 723000 Hanzhong, China
}

\begin{abstract}
In this paper, relations among the parameters of the non-minimum phase for the Buck-Boost converter are studied. It is demonstrated that when the Buck-Boost converter works in Buck mode, the negative regulation voltage is determined by the converter with the pre-designed inductor and load as the duty ratio $D<0.382$, as well as what is determined jointly by the converter with its pre-designed inductor, load and duty cycle as the duty ratio $D>0.382$, and the duty ratio has a limited effect on the negative regulation voltage of the converter; when the Buck-Boost converter works in Boost mode, the negative regulation voltage is determined jointly by the converter with its pre-designed inductor, load and duty cycle, for which performs that the higher the output voltage is(i.e., the greater the duty ratio is), the more severe undershoot of the voltage will be. The conclusion of this paper has a guidance meaning for decreasing the negative regulation voltage of the Buck-Boost converter. Finally the correctness of the analysis for the non-minimum phase is verified by simulations and experiments.
\end{abstract}

\section{Introduction}

Buck-Boost converter can easily implement a set of applications such as a step-up or stepdown of the voltage and a negative voltage output, etc., which is widely used in photovoltaic power generation system, new energy, aviation industry, aerospace technology and other fields. When Buck-Boost converter takes the capacitance voltage as its output for feedback control, it is a non-minimum system which is characterized by a zero point in the right half plane for the small-signal mathematical model. A notable feature of this zero point is that in the case of a sudden increase (or decrease) of the duty cycle, in the early stage, except the case that the output voltage is in overshoot, there will be a change that the voltage takes a rise after a drop(or a drop after a rise), which is namely the non-minimum phase response (negative voltage regulation process).Negative regulation process will deteriorate the dynamic quality of the control system, and extend the time for a transient process of the system. Furthermore, in the negative regulation stage, as the controller receives an error signal and then forms the positive feedback, which seriously affects the transient and steady state performance of the system [1-2].Therefore, to find out the relationship between the converter parameters and the course that leads to a non-minimum phase response will have a significant meaning for

ae-mail:jfhuang2000@163.com 
optimizing the performance of the converter design, and then further diminishes the influence of non-minimum phase response acting on the system, consequently improves the performance of the converter.

Buck-Boost converter has been extensively studied and has achieved many important results by far. By means of control strategies, deep studies on non-minimum phase response of the Buck-Boost converter are made in [3-6]; and the physical meaning of the zero in the right half plane for the Buck-Boost converter is analyzed in[7]. The impacts of the parameters including inductance, load and duty cycle on the non-minimum phase of the Buck-Boost converter have not been reported yet.In this paper, such effects of the inductance, load and duty cycle on the non-minimum phase of the Buck-Boost converter are analyzed in detail. The thesis points out that the smaller the inductance value is, the less the load is. For another aspect that the smaller the duty ratio is, the smaller the value of negative regulation load will be.

\section{Small-signal mathematical model of a Buck-Boost converter}

The circuit topology of a Buck-Boost converter is shown in Figure 1.

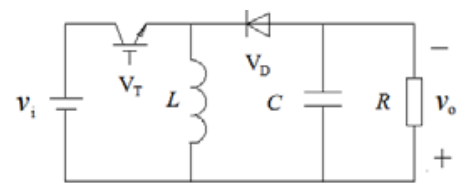

Figure 1. Buck-Boost converter

Buck-Boost converter works in the continuous conduction mode $(\mathrm{CCM})$ for controlling the design-based small signal mathematical model of the output voltage. The description shows in equation (1) can be found in[4].

$$
\frac{\hat{v}_{\mathrm{o}}(s)}{\hat{d}(s)}=\frac{V_{\mathrm{i}}\left(1-s D L / D^{\prime 2} R\right)}{s^{2} L C+s L / R+D^{\prime 2}}
$$

Through the analysis in equation (1), it can be seen that the mathematical model of a BuckBoost converter contains a zero in the right half plane, the zero is related to the converter design parameters. The relationship between the non-minimum phase response and the converter design parameters is then analyzed below in detail.

\section{Analysis of the relationship between non-minimum phase and the converter parameters}

From the analysis of equation(1), it can be seen that in the right half plane of the expression in a Laplace domain, the position of zero changes with the parameters of converter inductance, load and duty ratio. The changes of these parameters can cause a non-minimum phase response. In order to discuss the effect of these parameters on the non-minimum phase, a specific set of parameters of a converter is described. And if one of these parameters is changed, the other parameters would keep constant. Then draw positions for different poles and zeros in the root locus. According to the root locus and the results of circuit simulation, the impact of different component parameters in the same circuit on non-minimum phase of the system is then studied.

\subsection{Analysis of inductance response to non-minimum phase of the system}

The parameters of the Buck-Boost converter are presented as follows: the input voltage $V_{\mathrm{i}}=24 \mathrm{~V}$, capacitance $C=100 \mu \mathrm{F}$, load $R=8 \Omega$, duty cycle $D=0.4$ and the switching frequency $f_{\mathrm{s}}=50 \mathrm{kHz}$. The following work for the converter which works in CCM with different inductance $(L=500 \mu \mathrm{H}$ 、 
$2 \mathrm{mH} 、 5 \mathrm{mH})$ are discussed respectively. Simulation and analysis procedure is accomplished by using the power system simulation software PSIM6.0, simulation results are given to show the case that the non-minimum phase response presents for the output voltage when the system is operated stably with abrupt changes of duty cycle(at $30 \mathrm{~ms}$ the duty ratio jumps from 0.4 to 0.5 , and at $50 \mathrm{~ms}$ it jumps from 0.5 to 0.4 again).

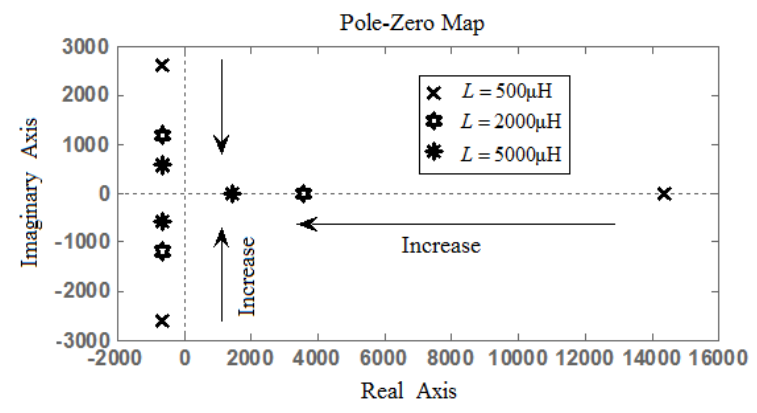

Figure 2. Root locus of zero and pole changes corresponding to different values of inductance

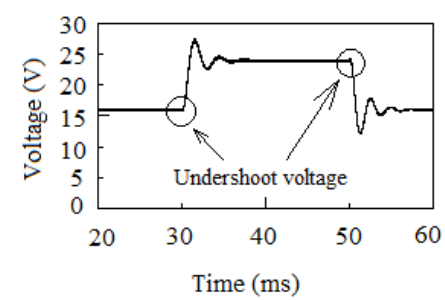

(a) $L=500 \mu \mathrm{H}$

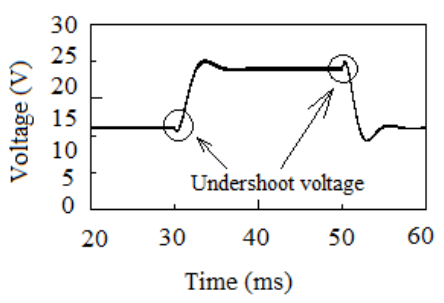

(b) $L=2 \mathrm{mH}$

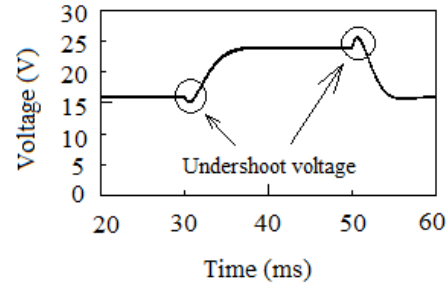

(c) $L=5 \mathrm{mH}$

Figure 3. The response for different inductance values with the corresponding non-minimum phase of the system

As can be known from the analysis of Figure 2, with an increase of the inductance from $500 \mu \mathrm{H}$ to $5 \mathrm{mH}$, the pole in the left half plane and the zero in the right half plane of S-domain are getting closer to the origin with the increase of inductance of the system. It can be seen from the simulation results in Figure 3, with the increased approximating of zero and pole to the origin, the non-minimum phase response of the system becomes more severe as the inductance increases, that is to say, when the duty cycle increases (or decreases), the output voltage does not immediately increase (or decrease), instead, it would first decrease (or increase) and then increases (or decreases) again, and with the increase of inductance, the negative regulation course would become more severe, with which is consistent with the features of non-minimum phase in the right half plane.

\subsection{Analysis of load response to non-minimum phase of the system}

The parameters of the Buck-Boost converter are presented as follows: the input voltage $V_{\mathrm{i}}=24 \mathrm{~V}$, capacitance $C=100 \mu \mathrm{F}$, inductance $L=500 \mu \mathrm{H}$, duty ratio $D=0.4$, and the switching frequency $f_{\mathrm{s}}=50 \mathrm{kHz}$. The following work for the converter which works in CCM with different resistance $(R=8 \Omega 、 5 \Omega 、 3 \Omega)$ are discussed respectively. Simulation results are given to show the case that the non-minimum phase response presents for the output voltage when the system is operated stably with abrupt changes of its duty cycle(at $30 \mathrm{~ms}$ the duty ratio jumps from 0.4 to 0.5 , and at $50 \mathrm{~ms}$ it jumps from 0.5 to 0.4 again).

As can be obtained from the analysis of Figure 4, with the load resistance decreasing from $8 \Omega$ to $3 \Omega$, the zeros of the system in the right half plane are getting close to the origin, as well as the poles of the system in the left half plane are getting close to the origin along the imaginary axis and is getting away from the origin along the real axis in an S plane. As can be obtained from the analysis of the simulation results in Figure 5, with the approximating process from the zeros to the origin, the 
negative regulation course becomes more significant, i.e., the negative regulation course is getting more severe while the load is getting heavier, this feature is consistent with the mathematical model of non-minimum phase characteristics.

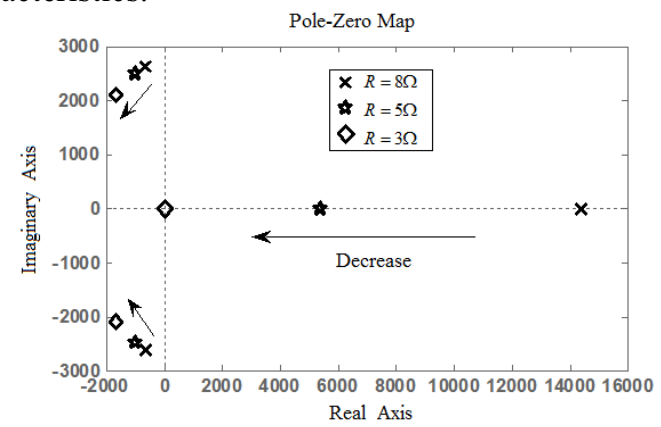

Figure 4. Root locus of zero and pole changes corresponding to different values of resistance

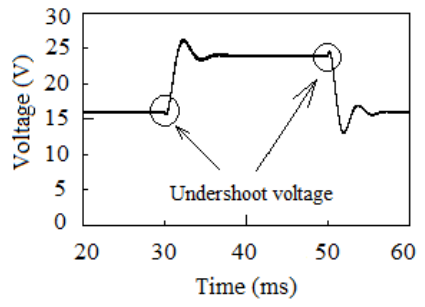

(a) $R=8 \Omega$

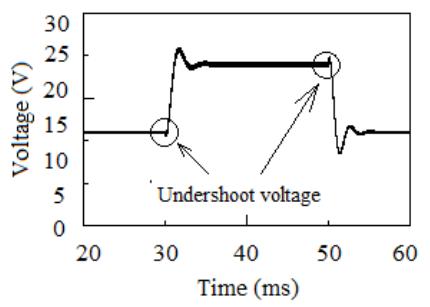

(b) $R=5 \Omega$

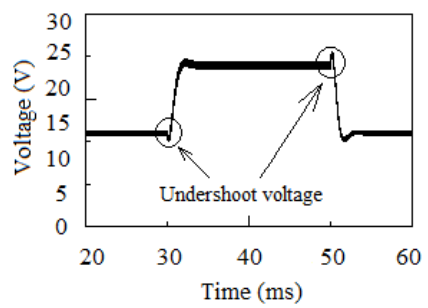

(c) $R=3 \Omega$

Figure 5. The response for different resistance values with the corresponding non-minimum phase of the system

\subsection{Analysis of duty cycle response to non-minimum phase of the system}

Transform equation (1) into equation (2), it can be obtained that

$$
\frac{\hat{v}_{\mathrm{o}}(s)}{\hat{d}(s)}=-\frac{V_{\mathrm{i}}(1-\lambda \cdot L s / R)}{L C s^{2}+L s / R+D^{\prime 2}}
$$

where $\frac{D}{D^{\prime 2}}=\lambda$, analysis of $\lambda$ we can get

$$
\frac{D}{D^{\prime 2}}=\frac{D}{(1-D)^{2}}=1
$$

As the simplification of equation (3), equation (4) is expressed as

$$
D^{2}-3 D+1=0
$$

Solve equation(4), then two solutions of the equation are obtained, where $D_{1}=0.382, D_{2}=2.618$ (abandoned),respectively. Namely when $D=0.382$, it can be determined that $\lambda=1$, therefore, the analysis course of non-minimum phase is divided into two parts by $D=0.382$ and to be discussed respectively. When $D<0.382$, it can be can be derived that $\lambda<1$, the non -minimum phase response is mainly determined jointly by the inductance and the load; and when $D>0.382$, it can be can be derived that $\lambda>1$, the non-minimum phase response is mainly determined jointly by the duty ratio, inductance and the load. The influence of duty ratio $D$ on $\lambda$ and $V_{\mathrm{o}}$ is shown in Table 1 .

Tab 1. The relationship between $D$ and $\lambda$ as well as that is between $\mathrm{D}$ and $V_{\mathrm{o}}$

\begin{tabular}{ccccccccccc}
\hline$D$ & 0.1 & 0.2 & 0.3 & 0.382 & 0.4 & 0.5 & 0.6 & 0.7 & 0.8 & 0.9 \\
\hline$\lambda$ & 0.13 & 0.31 & 0.61 & 1.0 & 1.11 & 2.5 & 3.75 & 7.78 & 20 & 90 \\
$V_{\mathrm{o}} / V_{\mathrm{i}}$ & 0.11 & 0.25 & 0.43 & 0.618 & 0.67 & 1.0 & 1.5 & 2.33 & 4 & 9 \\
\hline
\end{tabular}


When duty ratio $D<0.5$, the converter works in Buck mode. The analysis can be drawn from table 1 , and the analysis results can be divided into two cases, that is, when $D<0.382$, it can be can be derived that $\lambda<1$, the non-minimum phase response is mainly determined jointly by the duty ratio, inductance and the load, the duty cycle would weaken the negative regulation course which is caused by zeros in the right half plane at this point; when $0.382<D<0.5$, the non-minimum phase response is mainly determined jointly by the duty ratio, inductance and the load, the maximum value of the influence of the duty cycle on the negative regulation is 2.5 times than before. When the duty ratio $D>0.5$, the Buck-Boost converter works in Boost mode, and in this mode, the output voltage will increase as the duty ratio increases, however, the change of $\lambda$ is far more than the ratio of output to the input voltage, that is, the negative regulation course which is caused by the duty cycle becomes more severe, the significance of the negative regulation course will inevitably lead to a slow down for the system output response. The following work is to simulate and analyze the impact of the duty cycle on the non-minimum phase of the system. The parameters of the Buck-Boost converter are presented as follows: the input voltage $V_{\mathrm{i}}=24 \mathrm{~V}$, capacitance $C=100 \mu \mathrm{F}$, inductance $L=500 \mu \mathrm{H}$, load $R=8 \Omega$, and the switching frequency $f_{\mathrm{s}}=50 \mathrm{kHz}$. Then take a discussion in conditions of different duty ratios(as $D=0.5,0.6,0.7$, respectively), simulation results are given to show the case that the non-minimum phase response presents for the output voltage when the system is operated stably with abrupt changes of duty cycle(at $30 \mathrm{~ms}$ the duty ratio abruptly increases 0.1 , and at $50 \mathrm{~ms}$ it suddenly decreases 0.1 again).

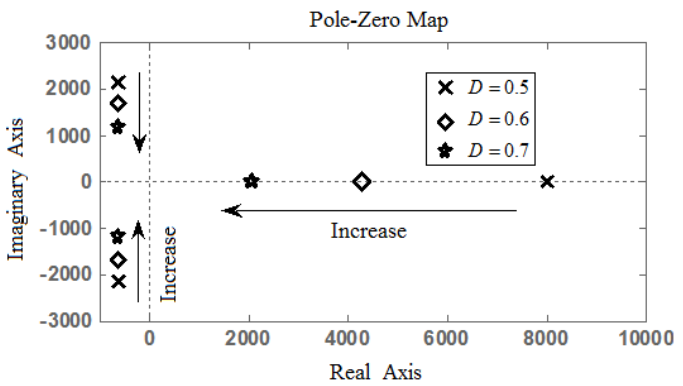

Figure 6. Root locus of zero and pole changes corresponding to different values of duty cycle

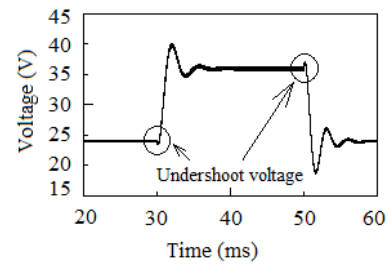

(a) $D=0.5$

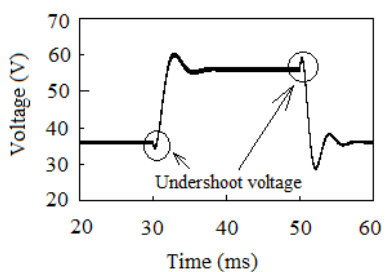

(b) $D=0.6$

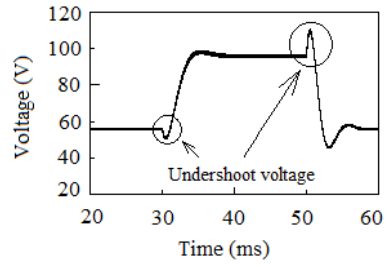

(d) $D=0.7$

Figure 7. The response for different duty cycle values with the corresponding non-minimum phase of the system

As can be known from the analysis of Figure 6, with an increase of the duty ratio from 0.5 to 0.7 , the pole in the left half plane and the zero in the right half plane of S-domain are getting closer to the origin with the increase of duty ratio of the system. It can be seen from the simulation results in Figure 7 , with the increased approximating of zero and pole to the origin, the negative regulation course of the system becomes more severe as the duty ratio increases, that is to say, the duty ratio has a great impact on the negative regulation course of the system. And it can be seen that in the simulation results, although the value of the inductance is much less that is barely $500 \mu \mathrm{H}$, the non-minimum phase response caused by the duty cycle is significant.

\section{Experimental tests}


In order to verify the correctness of the theoretical analysis for the non-minimum phase response of the Buck-Boost converter, an experimental platform is built for experimental verification. The parameters of the Buck-Boost converter are presented as follows: the input voltage $V_{\mathrm{i}}=24 \mathrm{~V}$, capacitance $C=470 \mu \mathrm{F}$, inductance $L=1 \mathrm{mH}$, the switching frequency $f_{\mathrm{s}}=50 \mathrm{kHz}$, and the duty ratios $D=0.4$ and 0.6 separately with a variation of the duty ratio $\Delta d=0.1$. The experimental results are shown in Figure 8.

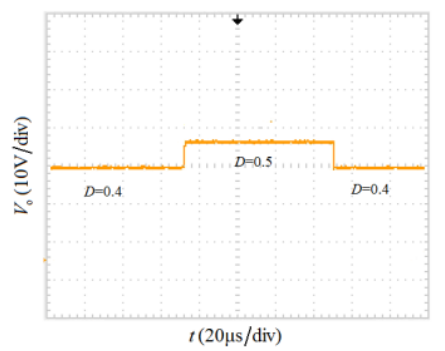

(a) $D=0.4$

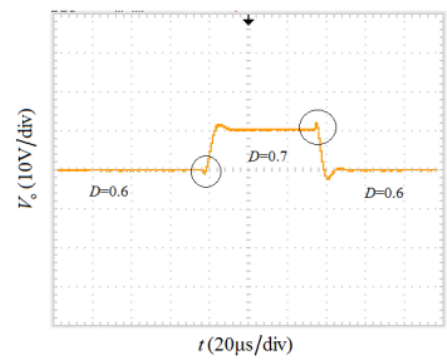

(b) $D=0.6$

Figure 8. The response for abrupt changes of the duty cycle with the corresponding non-minimum phase

The analysis for the experimental results are presented as follows: in Figure8(a), the duty ratio jumps suddenly from 0.4 to 0.5 , and then it abruptly jumps from 0.5 to 0.4 again while the inductance $L=1 \mathrm{mH}$. It can be seen from the experimental results that although the inductance value is higher than before, the negative regulation voltage presents a low state; in Figure8(b), the duty ratio jumps suddenly from 0.6 to 0.7 , and then it abruptly jumps from 0.7 to 0.6 again while the inductance $L=1 \mathrm{mH}$. It can be seen from the experimental results that the negative regulation voltage reaches about $2 \mathrm{~V}$, which indicates that the non-minimum phase response of the system will increase as the duty ratio increases.

\section{Conclusion}

The non-minimum phase response of a Buck-Boost converter which works in CCM is related to its inductance, load and duty ratio. When it works in Buck mode, the duty ratio has a limited effect on the non-minimum phase response of the system, which is mainly determined by the values of its inductance and loads. When it works in Boost mode, the non-minimum phase response of the system is not only to consider the impact of the inductance and the load, but also to consider the impact of the duty ratio on the system. With the increase of the output voltage, the non-minimum phase response becomes even more severe, i.e., the higher the output voltage is, the more severe undershoot of the voltage will be. The correctness of the theoretical analysis above is then verified by simulation and experiments. In this paper, the theoretical approach of analyzing the system for the negative regulation voltage of the nonminimum phase of Buck-Boost converter can be applied to other converter topologies, which has a guidance meaning for suppressing the undershoot voltage in a non-minimum phase system and improving the transient performance for the system.

\section{References}

1. C. Rodriguez, J. L. Guzman,Journal of Process Control 24, 368(2014)

2. H.H. Huang, C.L.Chen, Di.R.Wu,IEEE transactions on power electronics 27,354 (2012)

3. D.W.Hart,Introduction to power Electronics(prentice Hall,inc, 1997)

4. Z. Wu, H. Li, P. Zuo, and W. Z. Liu, Proceedings of the CSEE 22, 110(2001)

5. G. K. Schoneman,D. M. Mitchell, IEEE Trans. Power Electron. 3,31(1988)

6. J. Y. Le, Y. X. Xie, Q. Z. Hong, Z. Zhang, and L. Chen, Proceedings of the CSEE 31, 16(2011)

7. D. X. Shuai, Y. X. Xie, X. G. Wang, and F. X. Zhou, Proceedings of the CSEE 29, 15(2000) 